\title{
Evaluación de Cultivares de Maíz de Grano Amarillo en 9 Zonas Productoras de Panamá1
}

\author{
Daniel Pérez, Andrés González², Nivaldo De Gracia ${ }^{2}$, Ricardo Hernández ${ }^{2}$, Eric Quiróz ${ }^{2}$, \\ Ismael Camargo ${ }^{3}$, Alfonso Alvarado ${ }^{4}$
}

\section{COMPENDIO}

Se evaluaron híbridos y variedades del Programa Nacional de Panamá e híbridos comerciales y experimentales de compañías privadas que distribuyen semilla en el país, para determinar adaptación, rendimiento y principales características agronómicas. El estudio fue realizado por el Programa de Maíz del Instituto de Investigación Agropecuaria de Panamá, (IDIAP) en 9 localidades.

Del análisis combinado se encontró diferencias significativas entre las localidades, entre los cultivares y la interacción tratamiento x localidad, destacándose las localidades de Paraíso, Las Tablas y La Honda. En cuanto a los cultivares los mejores rendimientos se obtuvieron con el híbrido local P-8812 y los híbridos experimentales 477x372 y 477x375 obtuvieron rendimientos de 5089,5056 y $4915 \mathrm{~kg} / \mathrm{ha}$ respectivamente, superando al híbrido comercial X-3214 y experimental XL-604. La interacción muestra que la respuesta de algunos materiales varía de acuerdo a las condiciones de la localidad.

Los cultivares que resultaron con los mejores índices de estabilidad fueron Guararé-8128, XL-604, P-8802, TOC-80A, TOC-7428 y (477 x 304).

Palabras claves adicionales: híbridos, Zea mays L., estabilidad.

\section{INTRODUCCIÓN}

El Instituto de Investigación Agropecuaria de Panamá, en su programa de investigación en maíz, ha logrado desarrollar variedades de polinización abierta, adaptada a las condiciones ecológicas de las principales áreas productoras de maíz.

Las variedades liberadas por el IDIAP, no han sido adoptadas por la totalidad de los productores mecanizados y semimecanizados por considerar éstos que obtienen mejores resultados con los híbridos importados.

En 1986, con el apoyo del CIMMYT, se inician las evaluaciones de cruzas dialélicas, para determinar ACG y ACE, identificando híbridos dobles y triples predichos con rendimiento de grano superior y/o similar a los obtenidos con los híbridos simples de distribución comercial en el país.

Durante los años de 1987 y 1988, el programa evaluó en campos experimentales y fincas de productores los mejores híbridos predichos, identificando híbridos simples, triples y dobles con rendimiento y otras características agronó-

\begin{abstract}
$^{3}$
Maize varieties and hybrids from the Panama National l'rogram and experimental and commercial hybrids from priva te companies which distribute seed in Panama were evaluated in order to determine yield, agronomic characteristics and general adaptation. This study was conducted by the Maize Program of the Instituto de Investigacion Agropecuaria de Panama (IDIAP) at 9 different locations.

From the combined analysis, significant ditTerences were found between locations, cultivars and a significant cultivar by location interaction was present, with the locations Paraiso, Las Tablas and La Honda having much higher yields. The highest grain yields for the entries evaluated were obtained with hybrid P-8812 and experimental hybrids $477 \times 372$ and $477 \times 375$ (5.09,5.06 and $4.91 \mathrm{t} / \mathrm{ha}$ respectively). All three were superior to the commercial hybrids X-3214 and XL-604. The significant interaction between cultivar and location means that some of the materials evaluated have different responses by location.
\end{abstract}

The cultivares having improved stability parameters were Guarare8128, XL-604, P8802, TOC-890A and TOC-7428.

micas superiores a las del híbrido comercial X-304C, del cual se comercializan más de 200 toneladas de semilla por año.

En las actividades de 1989, en el ensayo uniforme de rendimiento sembrado en 9 localidades nuevamente fueron evaluados lo mejores híbridos y variedades del programa nacional, híbridos importados e híbridos desarrollados por el Programa Regional de Maíz de Centroamérica Panamá y El Caribe con los siguientes objetivos:

a) Comprobar el comportamiento de los mejores híbridos desarrollados por el IDIAP.

${ }^{1}$ Trabajo presentado en la XXXVI Reunión Anual del PCCMCA, San Salvador, El Salvador, C. A., marzo, 1990.

2 Investigadores del Programa de Maíz del Instituto de Investigación Agropecuaria de Panamá.

${ }^{3}$ Investigador responsable de producción de híbridos de maíz del Instituto de Investigación Agropecuaria de Panamá.

4 Jefe del programa de Maíz del Instituto de Investigación Agropecuario de Panamá.

${ }^{5}$ El abstract es traducción del compendio.

Publicado en Agronomía Mesoamericana, Vol. 2 (1991). 
b) Determinar los parámetros de estabilidad que permitan describir los genotipos de acuerdo a su respuesta a través de ambientes contrastes.

c) Permitir a los agricultores apreciar las características agronómicas favorables y rendimiento de grano de los mejores híbridos o variedades evaluados.

\section{REVISION DE LITERATURA}

Las pruebas de comportamiento de variedades cuando se realizan convencionalmente ofrecen información sobre la interacción genotipo-ambiente, pero no dan idea de la estabilidad de las variedades evaluadas (Córdova, 1978). De allí que el análisis de estabilidad es un buen instrumento en la identificación de germoplasma de gran potencial para los programas de mejoramiento.

Eberhart y Russel (1966) presentaron un modelo para determinar la estabilidad de variedades de maíz a través de can1bios ambientales, y definen una variedad estable como aquella con media de rendimiento alta, respuesta a índices ambientales de $\mathrm{B}_{\mathrm{i}}=1.0$ y con desviaciones de regresión $\left(\mathrm{S}_{\mathrm{di}}\right)$ tan pequeña como sea posible.

Varios investigadores Hallauer y Sears (1969) Ebehart (1971), y Darrah et al. (1972) han demostrado con resultados teóricos y empíricos que cuando se integran variedades de rendimiento similares en un complejo el promedio de rendimiento de éste es mayor que el promedio de las variedades progenitoras.

Velasquez et al. (1979) propone el método de mejoramiento paralelo que permite simultáneamente la formación de variedades de libre polinización e híbridos de maíz, en base a poblacionesmejoradas por hermanos completos.

\section{MATERIALES Y MÉTODOS}

La Prueba Regional de maíz del IDIAP, involucra la evaluación de cultivares del Programa Nacional, Programa de Centroamérica, Facultad de Ciencias Agropecuarias de Panamá, y Compañías Privadas Extranjeras, los cuales se presentan en el Cuadro 1. Estos fueron evaluados bajo un diseño de bloques completos al azar en 9 localidades de Panamá. La parcela experimental constó de dos surcos de 5 metros de largo, y la parcela útil es de 44 plantas, teóricamente.

Las variables estudiadas fueron: días a flor, altura de planta y mazorca, acame del tallo y de raíz, enfermedades importantes, número total y pudrición de mazorca y rendimiento de grano al $15 \%$ de humedad.

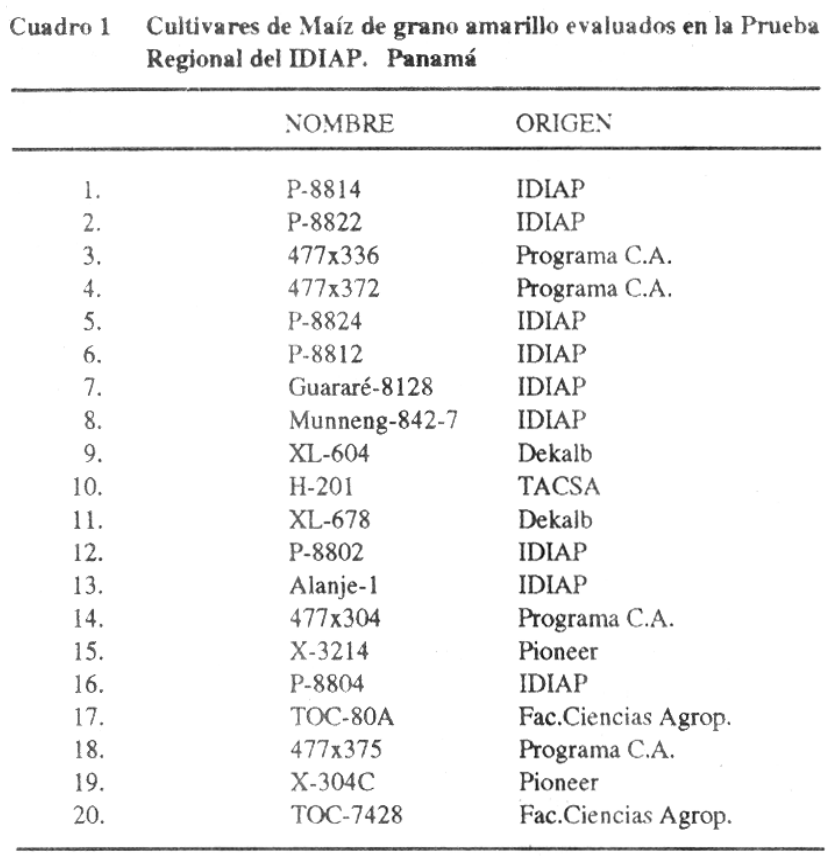

Cuadro 2 Características Agronómicas y Rendimiento de grano en kg / ha al $14 \%$ de humedad de Híbridos y variedades comerciales y experimetales de grano amarillo, evaluadas en seis localidades de la región de Azuero. Panamá 1989B.

\begin{tabular}{lcccccc}
\hline Entrada & $\begin{array}{c}\text { Rend. } \\
\mathrm{kg} / \mathrm{ha}\end{array}$ & $\begin{array}{c}\text { Días } \\
\text { a flor }\end{array}$ & $\begin{array}{c}\text { Altura } \\
\text { maz. }\end{array}$ & $\begin{array}{c}\text { Acame } \\
\text { tallo } \%\end{array}$ & $\begin{array}{c}\text { Pudrición } \\
\text { maz. \% }\end{array}$ & $\begin{array}{c}\text { \% sobare } \\
\text { X-3214 }\end{array}$ \\
\hline P-8812 & 5662 & 57 & 124 & 18.8 & 9.5 & 114 \\
$477 \times 375$ & 5379 & 56 & 116 & 18.3 & 9.5 & 108 \\
$477 \times 372$ & 5377 & 55 & 114 & 16.3 & 6.4 & 108 \\
XL-604 & 5189 & 58 & 119 & 18.1 & 9.2 & 105 \\
P-8814 & 5179 & 57 & 118 & 21.9 & 12.8 & 104 \\
P-8802 & 5070 & 56 & 114 & 18.5 & 10.9 & 102 \\
X-3214 & 4963 & 58 & 124 & 31.0 & 11.1 & $-\ldots$ \\
\hline
\end{tabular}

X Rendimiento: 4644 kg/ha; C.V.: 12.7\%; DMS : 303

Cuadro 3 Análisis de Varianza Combinado de 20 cultivares de Maíz en seis localidades

\begin{tabular}{lrrr}
\hline FUENTE DE & G L & C. M. & F.01 \\
VARIACION & & & \\
\hline LOCALIDAD & 5 & 19.97 & $* *$ \\
REP. (LOC) & 18 & 2.30 & \\
TRATAMIENTO & 19 & 8.67 & $* *$ \\
LOC*TRAT & 95 & 0.62 & $* *$ \\
\hline
\end{tabular}

\section{RESULTADOS Y DISCUSIÓN}

En el Cuadro 2, se presentan las medidas de las 
Cuadro 4 Características Agronómicas y Rendimiento de grano en $\mathrm{kg} /$ ha al $15 \%$ de humedad de Variedades e Híbridos de Maíz comerciales y experimentales en 9 localidades de Panamá, 1989-90.

\begin{tabular}{|c|c|c|c|c|c|c|c|c|c|}
\hline \multirow[b]{2}{*}{ ENTRADA } & \multirow{2}{*}{$\begin{array}{c}\text { Días a } \\
\text { flor }\end{array}$} & \multicolumn{2}{|c|}{ Altura (cms) } & \multirow{2}{*}{$\begin{array}{l}\text { Acame } \\
\text { tallo \% }\end{array}$} & \multirow{2}{*}{$\begin{array}{l}\text { No. Plantas } \\
\text { cosechadas }\end{array}$} & \multicolumn{2}{|c|}{ No.Mazorcas } & \multirow{2}{*}{$\begin{array}{l}\text { Cobertura } \\
\text { mazorca }\end{array}$} & \multirow{2}{*}{$\begin{array}{c}\text { Rendimiento } \\
\mathrm{kg} / \mathrm{ha}\end{array}$} \\
\hline & & planta & mazorca & & & totales & podridas & & \\
\hline P-8812 & 58 & 237 & 121 & 20.1 & 41 & 39 & 12.7 & 10.2 & 4958 \\
\hline $477 \times 372$ & 56 & 226 & 111 & 14.4 & 42 & 42 & 7.5 & 5.6 & 4951 \\
\hline $477 \times 375$ & 56 & 223 & 113 & 18.8 & 43 & 42 & 14.3 & 6.9 & 4818 \\
\hline P-8802 & 56 & 223 & 113 & 21.4 & 42 & 42 & 11.6 & 4.4 & 4586 \\
\hline XL-604 & 58 & 221 & 117 & 15.8 & 42 & 42 & 13.0 & 5.1 & 4585 \\
\hline P-8814 & 58 & 237 & 117 & 20.8 & 41 & 38 & 15.6 & 10.7 & 4573 \\
\hline$X-3214$ & 58 & 242 & 123 & 31.4 & 40 & 40 & 12.2 & 6.3 & 4542 \\
\hline P-8822 & 56 & 229 & 115 & 24.1 & 41 & 41 & 9.6 & 4.7 & 4528 \\
\hline P. 8824 & 56 & 227 & 117 & 19.8 & 42 & 41 & 10.4 & 7.2 & 4333 \\
\hline $477 \times 304$ & 55 & 211 & 103 & 16.5 & 42 & 41 & 7.6 & 3.8 & 4320 \\
\hline P- 8804 & 57 & 223 & 108 & 26.8 & 41 & 40 & 8.2 & 4.6 & 4137 \\
\hline TOC- 7428 & 57 & 235 & 122 & 19.9 & 41 & 38 & 13.1 & 5.4 & 4075 \\
\hline MUNENG-8428 & 56 & 220 & 108 & 14.5 & 41 & 39 & 10.9 & 7.1 & 3951 \\
\hline $477 \times 336$ & 58 & 218 & 108 & 16.9 & 40 & 37 & 13.0 & 15.5 & 3887 \\
\hline $\mathrm{X}-304 \mathrm{C}$ & 56 & 235 & 117 & 20.9 & 41 & 38 & 14.7 & 4.7 & 3840 \\
\hline ALANJE-1 & 57 & 228 & 120 & 23.0 & 40 & 37 & 10.5 & 4.1 & 3832 \\
\hline G- 8128 & 58 & 236 & 121 & 15.1 & 40 & 38 & 11.8 & 6.2 & 3762 \\
\hline XL-678 & 59 & 231 & 126 & 17.7 & 41 & 38 & 15.8 & 6.3 & 3730 \\
\hline TOC- $80 \mathrm{~A}$ & 57 & 234 & 119 & $=19.7$ & 40 & 35 & 14.7 & 5.2 & 3473 \\
\hline H-201 & 54 & 213 & 106 & 20.4 & 38 & 36 & 13.8 & 9.0 & 2968 \\
\hline $\mathrm{X}$ & 57 & 227 & 115 & 19.8 & 41 & 39 & 12.0 & 6.5 & 4192 \\
\hline
\end{tabular}

C.V. : $14.8 ;$ DMS. : 258

características agronómicas y rendimiento de grano obtenidas a través de 6 localidades de la Región de Azuero, principal productora de maíz de la República. Se puede apreciar que la mayor incidencia de acame de tallo y un alto porcentaje de pudrición de mazorca correspondió al híbrido comercial X-3214 que fue superado en rendimiento por 5 híbridos nacionales y 1 híbrido experimental de DEKALB (XL-604). La media de rendimiento varió de 3194 a $5662 \mathrm{~kg} / \mathrm{ha}$, correspondiendo los mejores rendimientos a los híbridos P-8812, 477x375 y $477 \times 372$ con 5662,5379 y $5377 \mathrm{~kg} /$ ha respectivamente.

En el cuadro 3 se presenta el análisis de varianza combinado de los cultivares a través de seis localidades, encontrándose diferencias altamente significativas para localidades, tratamiento y la interacción.

En el Cuadro 4 se presenta las medias de rendimiento y otras características agronómicas de los cultivares evaluados a través de 9 localidades. Los coeficientes de variación estimados fluctuaron desde 8.9 en Río Hato, hasta $20.5 \%$ en Las Tablas (Ver Cuadro 6), la mayoría de ellos estuvieron dentro del rango de confiabilidad. La media de rendimiento varió desde 2.97 (E-201) hasta 4.96 (P-88 12) t/ha. El mejor híbrido comercial X-3214 con rendimiento de 4.5 , t/ha, fue superado significativamente por los híbridos triples P-8812, $477 \times 372$ y $477 \times 375$ del programa nacional.

El análisis combinado de los 20 cultivares a través de nueve localidades se muestran en el cuadro 5, obteniéndose diferencias altamente significativas para tratamiento,
Cuadro 5 Análisis de varianza combinado de 20 cultivares en 9 localidades

\begin{tabular}{lrrr}
\hline FUENTE & G.L. & C.M. & F. 01 \\
\hline LOCALIDAD & 8 & 57.17 & $* *$ \\
REP (LOC) & 27 & 1.96 & \\
TRAT. & 19 & 9.88 & $* *$ \\
LOC*TRAT. & 152 & 0.67 & $* *$ \\
\hline
\end{tabular}

Cuadro 6 Medias de cinco características agronómicas de 9 cultivares evaluados en 9 localidades de Panamá. 1989-90.

\begin{tabular}{lcccccc}
\hline Entrada & $\begin{array}{c}\text { Rend. } \\
\mathrm{kg} / \mathrm{ha}\end{array}$ & $\begin{array}{c}\text { Días a } \\
\text { flor }\end{array}$ & $\begin{array}{c}\text { Altura } \\
\text { mazorca }\end{array}$ & $\begin{array}{c}\text { Acame } \\
\text { tallo \% }\end{array}$ & $\begin{array}{c}\text { Cobertura } \\
\text { maz. \% sobre }\end{array}$ & $\begin{array}{c}\text { X-304C } \\
\text { P-8812 }\end{array}$ \\
\hline $477 \times 372$ & 4958 & 58 & 121 & 19.0 & 9.7 & 129 \\
$477 \times 375$ & 4851 & 55 & 111 & 14.4 & 5.6 & 129 \\
P-8814 & 4573 & 56 & 113 & 18.8 & 6.8 & 125 \\
$477 \times 304$ & 4320 & 55 & 117 & 20.8 & 9.8 & 119 \\
$477 \times 336$ & 3887 & 58 & 108 & 16.5 & 3.6 & 113 \\
X-304 & 3840 & 56 & 117 & 21.0 & 4.3 & 100 \\
G-8128 & 3762 & 58 & 121 & 15.1 & 5.9 & 98 \\
H-201 & 2968 & 54 & 106 & 20.4 & 6.1 & 77 \\
\hline
\end{tabular}

X Rendimiento: $4287 \mathrm{~kg} / \mathrm{ha}$; C.V. : 11.6 ; 
localidades y tratamiento X localidades.

En el Cuadro 6, se incluyen algunas características agronómicas y rendimiento de grano de 9 cultivares del programa Nacional y de Centro América evaluados en 9 localidades comparados con los dos híbridos importados que más se siembran en el país. El híbrido comercial X- 304C, ampliamente sembrado en el país (200-250 toneladas de semilla importada anualmente) por los agricultores mecanizados, fue superado significativamente en rendimiento por 6 híbridos del programa nacional.

Las medias de rendimiento, las diferencias mínimas significativas y los coeficientes de variación obtenidos en las diferentes localidades se presentan en el cuadro 7. Los rendimientos más altos se obtuvieron en la localidad de Paraíso, y en La Honda, a pesar de que en esta última fue escasa la precipitación pluvial, además de la presencia de un período de estres, ocurrido 10 días antes de inicio de floración. Los datos de rendimiento y otras características agro-

Cuadro 7 Rendimiento promedio de 20 cultivares en 9 ambientes de Panamá. 1989

\begin{tabular}{lccl}
\hline Localidades & $\begin{array}{c}\text { Rendimiento } \\
\mathrm{kg} / \mathrm{ha}\end{array}$ & D.M.S. & C.V. \\
\hline PARTTA & 4531 & 988 & 15.4 \\
LA HONDA & 4792 & 668 & 10.7 \\
PARAISO & 5424 & 933 & 11.9 \\
GUARUMAL & 2803 & 745 & 18.8 \\
EL PITO & 3222 & 710 & 15.6 \\
LAS TABLAS & 4789 & 139 & 20.5 \\
GUARARE & 4243 & 692 & 11.5 \\
EL MANANTLAL & 4105 & 654 & 11.3 \\
\hline
\end{tabular}

Cuadro 8 Medias de seis características agronómicas de 9 cultivares evaluados en 12 localidades de Panamá. 1989-90.

\begin{tabular}{lcccccc}
\hline \multicolumn{1}{c}{ Entrada } & $\begin{array}{c}\text { Rend. } \\
\mathrm{kg} / \mathrm{ha}\end{array}$ & $\begin{array}{c}\text { Días a } \\
\text { flor }\end{array}$ & $\begin{array}{c}\text { Altura } \\
\text { Maz. }\end{array}$ & $\begin{array}{c}\text { Acame } \\
\text { tallo } \%\end{array}$ & $\begin{array}{c}\text { Cobertura } \\
\text { maz. \% }\end{array}$ & $\begin{array}{c}\% \text { sobre } \\
\text { X-304C }\end{array}$ \\
\hline $477 \times 375$ & 4957 & 56 & 112 & 19.9 & 6.9 & 127 \\
P-8812 & 4949 & 56 & 108 & 15.6 & 5.8 & 127 \\
$477 \times 372$ & 4866 & 58 & 112 & 23.1 & 9.6 & 124 \\
P-8814 & 4617 & 58 & 113 & 22.8 & 8.7 & 118 \\
$477 \times 304$ & 4287 & 55 & 101 & 18.9 & 5.2 & 109 \\
$477 \times 336$ & 4108 & 58 & 103 & 14.1 & 12.9 & 105 \\
X-304C & 3910 & 58 & 115 & 18.4 & 6.2 & 100 \\
G-8128 & 3877 & 55 & 111 & 17.2 & 6.4 & 99 \\
H-201 & 3013 & 54 & 105 & 25.4 & 5.6 & 77 \\
\hline
\end{tabular}

X Rendimiento: $4168 \mathrm{~kg} / \mathrm{ha}$; C.V. : 20.5 ;

nómicas, obtenidos mediante el análisis combinado para 12 localidades, se muestran en el Cuadro 8; en el mismo se puede apreciar que los híbridos nacionales superan en rendimiento a los híbridos importados. El X- 304C, fue superado en 27,27 y $24 \%$ de rendimiento por los híbrido8 $477 \times 375$, P-8812 y 477x372 respectivamente.
En el análisis de estabilidad (Cuadro 9) realizado para las 20 entradas a través de 9 localidades, los híbridos P-8802, 477x304 y XL-604 presentaron un mayor grado de estabilidad (Figura 1 y 2 ) además de alto rendimiento; las variedades de polinización abierta TOC- 7428 y TOC-80A mostraron buena estabilidad pero sus rendimientos fueron bajos.

El análisis combinado para 9 cultivares comunes a través de 12 localidades se presentan en el cuadro 10 , se observa que hay diferencias significativas para tratamiento, localidades y para la interacción.

Cuadro 9 Rendimiento, Coeficiente de Regresión y Desviaciones de Regresión de 20 entrada en 9 localidades. 1989.

\begin{tabular}{lccc}
\hline Entrada & $\mathrm{kg} / \mathrm{ha}$ & $\mathrm{B}_{\mathrm{i}}$ & $\mathrm{S}_{\mathrm{di}}$ \\
\hline & & & \\
P-8812 & 4958 & 1.331 & 0.168 \\
$477 \times 372$ & 4951 & 0.852 & 0.118 \\
$477 \times 375$ & 4817 & 1.162 & 0.079 \\
P-8802 & 4663 & 1.084 & 0.116 \\
XL-604 & 4584 & 1.165 & 0.149 \\
P-8814 & 4573 & 1.358 & 0.119 \\
P-8822 & 4528 & 0.928 & 0.163 \\
X-3214 & 4430 & 1.423 & 0.263 \\
P-8824 & 4333 & 0.939 & 0.125 \\
$477 \times 304$ & 4319 & 0.999 & 0.085 \\
P-8804 & 4137 & 0.935 & 0.230 \\
TOC-7428 & 4019 & 1.076 & 0.204 \\
MUNNENG-8227 & 3951 & 0.851 & 0.193 \\
$477 \times 336$ & 3887 & 0.698 & 0.413 \\
X-304C & 3840 & 0.766 & 0.132 \\
ALANJE-1 & 3832 & 0.892 & 0.109 \\
G-8128 & 762 & 1.065 & 0.066 \\
XL-678 & 3730 & 1.208 & 0.183 \\
TOC-80A & 3473 & 1.056 & 0.117 \\
H-201 & 2968 & 0.661 & 0.177 \\
\hline
\end{tabular}

Cuadro 10 Análisis de varianza combinado de: 9 variedades en 12 localidades.

\begin{tabular}{lrrr}
\hline \multicolumn{1}{c}{ FUENTE } & G.L. & C.M. & F.01 \\
\hline LOCALIDAD & 11 & 24.32 & $* *$ \\
REP (LOC) & 36 & 1.17 & \\
TRAT. & 8 & 19.76 & $* *$ \\
LOC*TRAT. & 88 & 0.73 & $* *$ \\
\hline
\end{tabular}

\section{CONCLUSIONES}

1. Los híbridos triples del programa nacional P-8812 (477x375) y (477x372), evaluados en 6 localidades de la Región de Azuero, mostraron superioridad en rendimiento y otras características agronómicas sobre elhíbrido comercial X-3214.

2. Los híbridos triples P-8812, (477x372) y (477x375), 


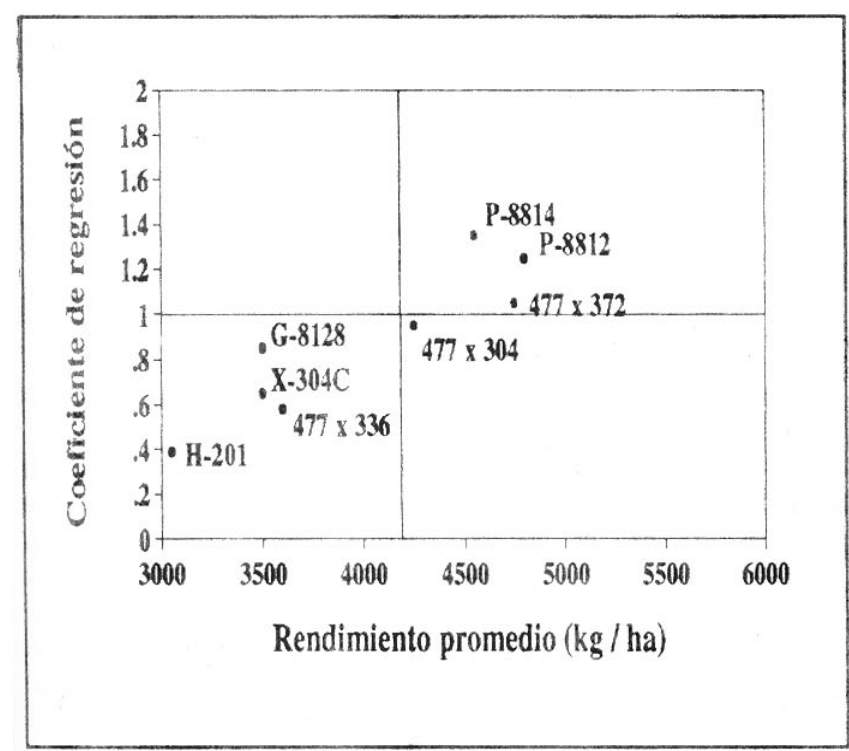

Figura 1 Relación entre adaptación (coeficiente de regresión) y el rendimiento pormedio de 9 entrada en 12 localidades de Panamá.

superaron en rendimiento a los híbridos comerciales X3214, X-304C y H-201, evaluados en 9 localidades de las provincias de Herrera, Los Santos, Coclé y Veraguas.

3. Los híbridos triples (477x375) P-8812, (477x372) y P-8814, comunes para la prueba regional y la evaluación de híbridos nacionales, realizada en 9 localidades del país, nuevamente mostraron superioridad sobre los híbridos importados X-304C y H-201.

4. Los híbridos P-8802 y (477x304), mostraron un mayor grado de estabilidad a través de las 9 localidades, evaluadas, además de presentar altos rendimientos.

\section{RECOMENDACIONES}

1. Liberar para uso comercial los híbridos triples del programa nacional P-8812 y P-8814, dada su estabilidad en diversidad de ambientes y altos rendimientos.

2. Continuar con la evaluación de los híbridos triples (477x304), (477x372), (477x375), yaque los mismos mostraron superioridad en rendimiento y otras características agronómicas sobre los híbridos comerciales X-304C y H-201.

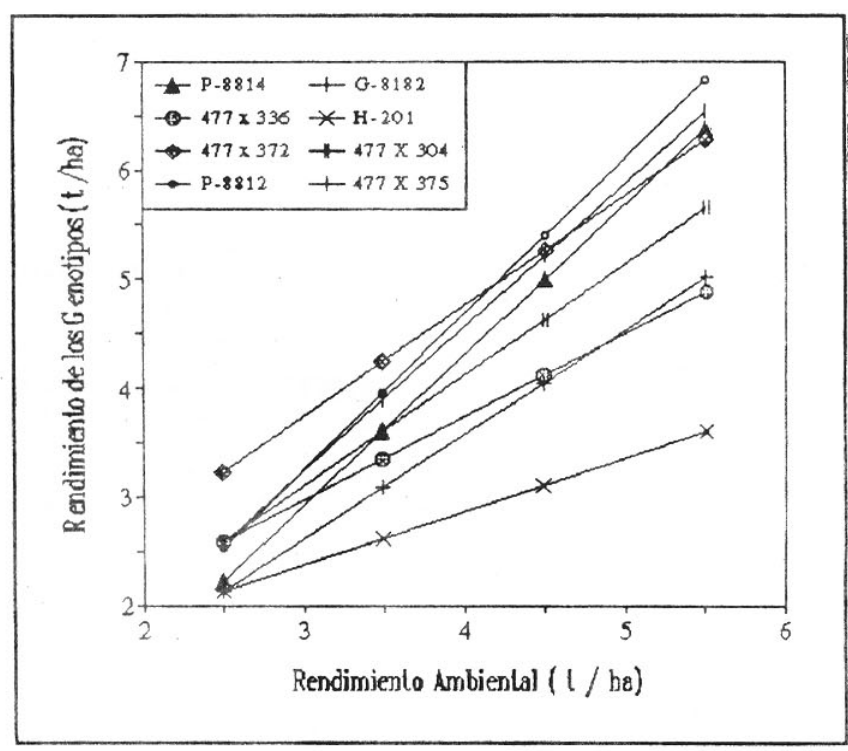

Figura 2 Líneas de Regresión de 9 variedades evaluadas en 9 localidades en Panamá.

\section{BIBLIOGRAFIA}

CARBALLO. C. A. y MARQUEZ, S. P. 1970. Comparación de variedades de maíz de El Bajio y La Mesa Central por su rendimiento y estabilidad. Agro-Ciencias 5(1): 129-146.

CORDOV A. H. S. 1978. Uso de parámetros de estabilidad para evaluar el comportamiento de variedades. Guatemala, ICTA $35 \mathrm{p}$.

DA RRAH L. L. S. A.. EBERHART; L. H. PENNY. 1972. A maize breeding metbods study in Kenya. Crop Sci. 12:605-608.

EBERHART. S. A. and W. A. RUSSEL. 1966. Stability parameters for comparing varieties. Crop. Sci. 6:36-40.

EBERHART, S. A. 1971. Regional maize dialles with U.S. and semi-exotic varieties. Crop Sci. 11:911-914.

HALLAUER. A. R.; J. H. SEARS. 1968. Second phase in tbe Evaluation of syntbetic varieties of maize for yield. Crop Sci. $8: 448-451$.

VELASQUEZ, R., CORDOV A, H. y POEY, F. 1979. Selección de hermanos completos por el método perse y mestizos para la formación de híbridos de maíz (Zea mays L.) XXV Reunión Anual del PCCMCA. Tegucigalpa, Honduras. 